\title{
Initial-state, mean-free-path, and skin-depth dependence of attosecond time-resolved IR-streaked XUV photoemission from single-crystalline magnesium
}

\author{
Q. Liao and U. Thumm \\ Department of Physics, Kansas State University, Manhattan, Kansas 66506, USA
}

(Received 12 February 2014; published 25 March 2014)

\begin{abstract}
We analyze the dependence of attosecond streaked photoelectron spectra and photoemission time delays from valence band (VB) and $2 p$ core-level (CL) states of a single-crystalline $\operatorname{Mg}(0001)$ thin film on the (i) modeling of the substrate electronic structure, (ii) electron mean free path (MFP), (iii) screening of the near-infrared (NIR) streaking laser field, and (iv) chirp of the attosecond extreme ultraviolet (XUV) pulse. Our quantum-mechanical numerical simulations predict streaked photoemission spectra that depend sensitively on the XUV chirp and weakly on the screening of the streaking laser field by the substrate. They furthermore show that streaking time delays for VB emission are relatively insensitive to the modeling of the initial quantum states, electron MFP, and NIR skin depth of the Mg substrate, in contrast to the stronger dependence of streaking time delays for $2 p$ CL emission on these factors.
\end{abstract}

DOI: 10.1103/PhysRevA.89.033849

PACS number(s): 42.50.Hz, 42.65.Re, 79.60.-i

\section{INTRODUCTION}

The time scale for the electronic motion in matter is typically one atomic unit $\left(1\right.$ a.u. $=24$ as $\left.=24 \times 10^{-18} \mathrm{~s}\right)$. During the past decade, table-top laser systems have become available for the attosecond (as) time-resolved investigation of the ultrafast electronic dynamics in atoms [1,2] and solid surfaces $[3,4]$. The dominant enabling technique for such attosecond experiments employs streaked photoelectron (PE) spectroscopy. In this technique, an attosecond extreme ultraviolet (XUV) pulse is employed to photoemit electrons into the electric field of a synchronous delayed femtosecond (fs) near-infrared (NIR) laser pulse [5]. Variation of the relative time delay $\tau$ between the XUV and NIR pulses allows for the measurement of "NIR-streaked" PE spectra. In these spectra XUV PE emission from a particular state of the target atom or surface is mapped to a "streaking trace", i.e., an oscillatory change of the recorded PE kinetic energy as a function of $\tau$ with the period of the NIR laser pulse. Thus streaked PE spectra yield both temporal and spectral information on emitted PE wave packets [6-8]. By comparing the temporal (i.e., phase) shift of the streaking traces for photoemission from two initial states with different binding energies, an apparent relative photoemission delay $\Delta \tau$ can be determined by analyzing a single streaked PE spectrum. This is usually achieved by first spectrally averaging individual streaking traces, so that $\Delta \tau$ can be obtained as the temporal shift between the centers of energy of the two streaking traces. With this method, apparent relative photoemission delays for the streaked XUV emission from different levels of isolated atoms in the gas phase [1] and energy bands in solid surfaces [3,4] of the order of several tens of attoseconds [1,3] or less [4] have been measured.

While this state-of-the-art experimental technique can explore ultrafast electronic dynamics in atoms and solids on the attosecond time scale, the exact theoretical description and thorough understanding of the laser-assisted XUVphotoionization process is challenging and has been a subject of significant controversy over the past few years. Several independent theoretical approaches [1,9-13], that are based on the time-dependent Schrödinger equation (TDSE) and include multielectron correlation at various levels of approximation, all significantly underestimate the measured relative delay $\Delta \tau_{2 s-2 p}=21 \pm 5$ as [1] between photoemission from $2 s$ and $2 p$ levels of isolated neon atoms.

The exact quantitative description of photoemission from solid surfaces is more complicated than for isolated atoms due to their complex electronic band structure $[3,14,15]$, elastic and inelastic collisions of photoreleased electrons inside the solid [16-18], the screening of the NIR streaking field in the solid [17,18], and surface and bulk plasmon excitations [19,20]. In the TDSE approach of Kazansky and Echenique [17], the valence-band (VB) initial states are modeled in terms of a "Chulkov potential" [21] and the corelevel (CL) initial states by combined Chulkov and Yukawa potentials. Chulkov potentials are effective single-activeelectron potentials that are adjusted to density-functional calculations and able to reproduce the measured valence-band electronic structure of solid surfaces [21]. The combination of Chulkov and Yukawa potentials provides sufficient flexibility to reproduce the CL electronic structure of solids at reasonable accuracy, with enhanced wave-function amplitudes at the surface atomic layer for the energetically highest levels within the narrow CL bands [22]. More elementary single-activeelectron-approximation (SAE) representations for the initial states in the streaked XUV photoemission from metal surfaces employ a "jellium" potential for the VB [23,24] and isolated atomic wave functions localized at the lattice points for the narrow CL bands ("zero-bandwidth model") $[18,25]$. In the jellium approach, the valence electronic spectrum of metal surfaces is modeled by a parametrized potential, and the attractive force of the positive lattice-centered ionic cores on the SAE is averaged to yield a spatially homogenous positive charge background inside the solid [23].

Even though the modeling of the surface electronic structure in terms of Chulkov and Yukawa potentials is expected to be more accurate than the use of jellium and separated single-atom potentials centered at the lattice points of the substrate, both approaches $[17,18]$ could explain the measured large relative delay $\Delta \tau_{V B-4 f}=110 \pm 70$ as between photoemission from $\mathrm{VB}$ and $4 f$ CL band electrons from $\mathrm{W}(110)$ surfaces [3]. In calculations with energy-independent electron 
mean free paths (MFPs), this appeared to provide evidence for the very different degree of spatial localization of initial VB and CL wave functions being a major determinant of the relative photoemission time delay, while other characteristics of VB and CL appeared to matter comparatively little [26].

Interestingly, however, while the facts that CL wave functions are localized at the lattice points and VB wave functions tend to be delocalized over the entire substrate cooperate with the measured relative delay $\Delta \tau_{V B-4 f}$ for $\mathrm{W}(110)$ surfaces, the interpretation of relative time delays in surface photoemission in terms of initial-wave-function-localization characters was very recently challenged by streaked photoemission experiments of Neppl et al. [4,27] with $\mathrm{Mg}(0001)$ surfaces. Even though magnesium more closely resembles an ideal free-electron metal (with completely delocalized VB wave functions and a large difference in CL and VB wave-functionlocalization character) than tungsten, Neppl et al. measured a much smaller relative delay of only $\Delta \tau_{V B-2 p}=5 \pm 20$ as between the streaked XUV photoemission from the VB and $2 p$ CL band of a $\mathrm{Mg}(0001)$ surface than the relative delay of $110 \pm 70$ as reported earlier by Cavalieri et al. [3] for W(110). Within a one-dimensional TDSE approach, modeling the initial electronic states in terms of SAE Chulkov and Yukawa potentials, Borisov et al. [28] were able to reconcile this discrepancy. By adjusting the contributions of resonant interband and nonresonant surface photoemission, they showed that their model can reproduce the relative photoemission delays $\Delta \tau_{V B-4 f}$ and $\Delta \tau_{V B-2 p}$ measured on $\mathrm{W}(110)$ and $\mathrm{Mg}(0001)$ surfaces, respectively. In particular, they found that resonant interband transitions of $\mathrm{VB}$ electrons reduce $\Delta \tau_{V B-2 p}$ and predicted $\Delta \tau_{V B-2 p} \approx 16$ as assuming dominant resonant transitions.

In an independent approach, within an improved strongfield-approximation (SFA) model, employing Chulkov and Yukawa potentials to represent the initial quantum state in the photoemission process, realistic energy-dependent MFP values, and a small adjusted value for the NIR skin depth, we recently reproduced the value of $\Delta \tau_{V B-2 p}$ measured by Neppl et al. [22]. Our quantum-mechanical results indicate that the $2 p$ photoemission delay is sensitive to both, MFP and NIR skin depth, while the VB photoemission delay is almost independent of the MFP and skin depth. This is in conflict with a classical heuristic model, which assumes that the streaking field disappears inside the metal (vanishing skin depth) and interprets accumulated photoemission time delays as the average travel time of PEs over a distance to the surface equal to the electronic MFP $[3,4]$.

Streaked photoemission spectra of metal surfaces and, in particular, relative photoemission delays, are sensitive observable quantities that are influenced by (i) details of the complex interactions of the XUV and NIR pulse with the substrate, (ii) material properties (such as the XUV and NIR dielectric response and elastic and inelastic electron scattering), and (iii) the dispersion of the released PE wave packet. In this work we thus significantly extend our previous study of photoemission from metal surfaces [22] and present a systematic investigation of the dependence of streaked photoemission spectra and relative photoemission delays on the modeling of the quantum-mechanical initial states, the chirp of the XUV attosecond pulse, the electronic MFP, and the screening of the streaking laser field. We organized this paper in the following way. Following this introduction, we discuss our numerical model in Sec. II. We subdivided Sec. II into a description of our general framework for the calculation of photoemission yields (Sec. II) and our modeling of VB initial states (Sec. II B) and CL initial states (Sec. II C). Section III contains our numerical results for photoemission from VB states (Sec. III A), CL states (Sec. IIIB), and relative photoemission delays, $\Delta \tau_{V B-2 p}$, for single-crystalline magnesium (Sec. III C). Our conclusion follows in Sec. IV. We use atomic units and relate electronic levels and PE kinetic energies to the Fermi level throughout this work unless stated otherwise.

\section{NUMERICAL MODEL}

\section{A. Transition amplitude for streaked photoemission}

We employ a quantum-mechanical approach based on the SFA to calculate streaked photoemission spectra from metal surfaces $[18,22]$. In this approach the electron is liberated from an initial Bloch state $\Psi_{\mathbf{k}_{i}}(\mathbf{r}, t)$ [29] into a NIR field-dressed final continuum state $\Psi_{\mathbf{k}_{f}}(\mathbf{r}, t)$ by absorbing a single XUV photon. The photoemission transition amplitude in the dipole-length gauge and to first order in the electric field $E_{X}$ of the XUV pulse is then given by

$$
T_{\mathbf{k}_{f}, \mathbf{k}_{i}}(\tau)=-i \int d t E_{X}(t)\left\langle\Psi_{\mathbf{k}_{\mathrm{f}}}(\mathbf{r}, t)|z| \Psi_{\mathbf{k}_{i}}(\mathbf{r}, t)\right\rangle .
$$

We assume the linear polarization direction of the XUV and IR pulses to coincide with the surface normal, approximating grazing incidence of both pulses in the experiment $[3,4]$ and associate the surface normal with the $z$ axis of our coordinate system.

The dipole-length-gauge interaction emphasizes large distances [30]. It is thus appropriate for large distances $z>0$ on the vacuum side of the metal-vacuum interface where our approximate representation of the true final-state wave function in terms of a "Volkov" wave function (see below) is more accurate than for small distances [31]. Our use of the dipole-length gauge is furthermore motivated by length-gauge SFA calculations for atomic ionization processes matching exact numerical calculations [32]. Regardless of the particular gauge used, the dipole approximation is applicable inside the solid for two reasons. First, even though the wavelength of the XUV pulse is comparable to the thickness of the $\operatorname{Mg}(0001)$ film in our numerical applications in Sec. III, contributions to the transition amplitude are effectively limited to small distances $z<0$ inside the solid. This is due to our use of physically motivated damping factors in the final PE states (see below). The second reason is linked to the XUV wave vector $\mathbf{k}_{X}$ being perpendicular to the surface normal $\left(\mathbf{e}_{z}\right)$ in our numerical applications such that the exponential $e^{i \mathbf{k}_{X} \cdot \mathbf{e}_{z}}=1$, while the breakdown of the dipole approximation would require $e^{i \mathbf{k}_{X} \cdot \mathbf{e}_{z}} \neq 1$ [33].

Assuming translation invariance of the substrate-electronic structure in the surface $(x-y)$ plane, we write the VB and CL initial-state wave functions as

$$
\Psi_{\mathbf{k}_{i}}(\mathbf{r}, t)=(2 \pi)^{-1} e^{i \mathbf{k}_{i, \|} \mathbf{r}_{\|}} \psi_{i}(z) e^{-i \varepsilon_{i} t}
$$


$\mathbf{k}_{i, \|}$ and $\mathbf{r}_{\|}$designate projections of the crystal momentum $\mathbf{k}_{i}=\left(\mathbf{k}_{i, \|}, k_{i, z}\right)$ and position vector $\mathbf{r}=\left(\mathbf{r}_{\|}, z\right)$ onto the surface plane, respectively, and $\varepsilon_{i}$ the binding energy of the initial state.

We model the final PE state in SFA as a damped Volkov wave function [18,22] with final momentum $\mathbf{k}_{f}=\left(\mathbf{k}_{f, \|}, k_{f, z}\right)$,

$$
\Psi_{\mathbf{k}_{f}}(\mathbf{r}, t)=(2 \pi)^{-3 / 2} f(\lambda ; z) e^{i \mathbf{p} \cdot \mathbf{r}} e^{i \phi_{k_{f, z}}(z, t)} .
$$

The damping factor

$$
f(\lambda ; z)=\Theta\left(z-z_{i}\right)+\Theta\left[-\left(z-z_{i}\right)\right] e^{\left(z-z_{i}\right) /(2 \lambda)}
$$

is related to the metal-vacuum-interface position $z_{i}$ and electron MFP $\lambda$. The parameter $\lambda$ accounts for elastic and inelastic electronic collisions during the propagation of the PE inside the solid. $\Theta$ is the unit step function. $\mathbf{p}_{\|}=\mathbf{k}_{f, \|}$ is the surface projection of the electron initial kinematic momentum $\mathbf{p}=\left(\mathbf{p}_{\|}, p_{z}\right)$ and $E=k_{f, z}^{2} / 2$ the final kinetic energy of the PE. Under these assumptions (1) becomes

$$
\begin{aligned}
T_{\mathbf{k}_{f}, \mathbf{k}_{i}}(\tau)= & -i \sqrt{2 \pi} \delta^{(2)}\left(\mathbf{k}_{i, \|}-\mathbf{k}_{f, \|}\right) \\
& \times \int d t E_{X}(t) e^{-i \varepsilon_{i} t} D_{i}(\lambda, t, \tau),
\end{aligned}
$$

with the dipole matrix element

$D_{i}(\lambda, t, \tau)=\int d z f(\lambda ; z) e^{-i \phi_{k f, z}(z, t, \tau)} e^{-i p_{z}(z, t, \tau) z} z \psi_{i}(z)$.

The nonperturbative interaction of the released PE with the NIR field $E_{L}(z, t)$ is taken into account in the $z$-dependent Volkov phase $\phi_{k_{f, z}}$. In a semiclassical approach, we calculate $\phi_{k_{f, z}}$ approximately, based on classical PE trajectories $\tilde{z}\left(t^{\prime}, \tau\right)$ with initial positions $\tilde{z}(t, \tau)=z$ and initial momenta

$$
p_{z}(z, t, \tau)=k_{f, z}+\int_{t}^{\infty} d t^{\prime} E_{L}\left[\tilde{z}\left(t^{\prime}, \tau\right), t^{\prime}-\tau\right]
$$

at the ionization times $t$. This yields

$$
\phi_{k_{f, z}}(z, t, \tau)=\frac{1}{2} \int_{t}^{\infty} d t^{\prime} p_{z}\left(z, t^{\prime}, \tau\right)^{2}
$$

where

$$
\begin{aligned}
p_{z}\left(z, t^{\prime}, \tau\right) & =p_{z}(z, t, \tau)-\int_{t}^{t^{\prime}} d t^{\prime \prime} E_{L}\left[\hat{z}\left(t^{\prime \prime}, \tau\right), t^{\prime \prime}-\tau\right], \\
\hat{z}\left(t^{\prime}, \tau\right) & =z+\int_{t}^{t^{\prime}} d t^{\prime \prime} p_{z}\left(z, t^{\prime \prime}, \tau\right)
\end{aligned}
$$

follow from Newton's equations for the classical PE motion.

The streaked photoemission probability is obtained by incoherently summing over all occupied initial states,

$$
P(E, \tau)=\sum_{i}\left|T_{\mathbf{k}_{f}, \mathbf{k}_{i}}(\tau)\right|^{2} .
$$

The center of energy (COE) as a function of $\tau$ is calculated by averaging $P(E, \tau)$ over the spectral range of a given streaking trace according to $E_{\mathrm{COE}}(\tau)=\int d E E P(E, \tau) / \int d E P(E, \tau)$.

We use the laser parameters of the experiment [4]. The XUV pulse,

$$
E_{X}(t)=E_{X, 0} e^{-2 \ln 2\left(t / \tau_{X}\right)^{2}} e^{-i\left(\omega_{X} t+\beta t^{2}\right)},
$$

has the pulse length $\tau_{X}=435$ as, photon energy $\hbar \omega_{X}=$ $118 \mathrm{eV}$, and linear chirp rate $\beta$. The NIR pulse electric field $E_{L}=-\partial A_{L} / \partial t$ is assumed to be screened at the surface, with skin depth $\delta_{L}$, and to decay exponentially inside the solid, starting at the metal-vacuum interface, according to the damping function

$$
g\left(\delta_{L} ; z\right)=\Theta\left(z-z_{i}\right)+\Theta\left[-\left(z-z_{i}\right)\right] e^{\left(z-z_{i}\right) / \delta_{L}} .
$$

The position-dependent screened NIR pulse is thus given by

$$
E_{L}(z, \hat{t})=g\left(\delta_{L} ; z\right) E_{L}(\hat{t}),
$$

with

$$
E_{L}(\hat{t})=E_{L, 0} e^{-2 \ln 2\left(\hat{t} / \tau_{L}\right)^{2}} \cos \left(\omega_{L} \hat{t}+\varphi\right) .
$$

$E_{L, 0}$ corresponds to the peak intensity of the NIR pulse and $\hat{t}=t-\tau . \varphi$ is the carrier-envelope phase (CEP), $\tau_{L}=5 \mathrm{fs}$, and $\hbar \omega_{L}=1.5 \mathrm{eV}$. For delays $\tau>0$ between the centers of the XUV and NIR pulse envelopes, the XUV pulse precedes the NIR pulse.

\section{B. Emission from valence-band states}

\section{Jellium model}

We consider a row of 31 magnesium atoms separated by the $\operatorname{Mg}(0001)$ interlayer spacing $a_{s}=4.923=2.6 \AA$ [21]. In the jellium model, the SAE potential for $\mathrm{VB}$ electrons is given by [34]

$$
U(z)= \begin{cases}-U_{0}, & |z| \leqslant d / 2, \\ 0, & |z|>d / 2,\end{cases}
$$

where $d$ is the thickness of the $\mathrm{Mg}$ slab and $U_{0}=\varepsilon_{F}+W$. The Fermi energy is $\varepsilon_{F}=7.08 \mathrm{eV}$ [29] and the work function $W=3.66 \mathrm{eV}$ [35]. The metal-vacuum-interface positions are defined at $\left|z_{i}\right|=d / 2$. The completely delocalized VB states are obtained by diagonalizing the corresponding Hamiltonian, $-\partial^{2} /\left(2 \partial z^{2}\right)+U(z)$. This results in 34 occupied states below the Fermi level that cover a bandwidth of $\sim 7 \mathrm{eV}$. Of these, the lowest and highest occupied jellium states are shown in Fig. 1.

\section{Chulkov model} form

The Chulkov potential [21] has the general parametrized

$$
U_{C}(z)=U_{1}(z)+U_{2}(z)+U_{3}(z)+U_{4}(z),
$$

where

$$
\begin{aligned}
U_{1}(z) & =A_{10}+A_{1} \cos \left[\frac{2 \pi}{a_{s}}\left(z-\frac{d-a_{s}}{2}\right)\right], \\
0 & \leqslant z \leqslant \frac{d-a_{s}}{2}, \\
U_{2}(z) & =-A_{20}+A_{2} \cos \left[\beta^{\prime} z\right], \quad \frac{d-a_{s}}{2}<z \leqslant z_{1}, \\
U_{3}(z) & =A_{3} \exp \left[-\alpha\left(z-z_{1}\right)\right], \quad z_{1}<z \leqslant z_{i m} \\
U_{4}(z) & =27.21 \mathrm{eV} \frac{\exp \left[-\lambda^{\prime}\left(z-z_{i m}\right)\right]-1}{4\left(z-z_{i m}\right)}, \quad z>z_{i m} .
\end{aligned}
$$

It is symmetric with respect to the center of the magnesium slab at $z=0$ (Fig. 2). Fitting this functional form to the numerical results of pseudopotential local-density-functional 

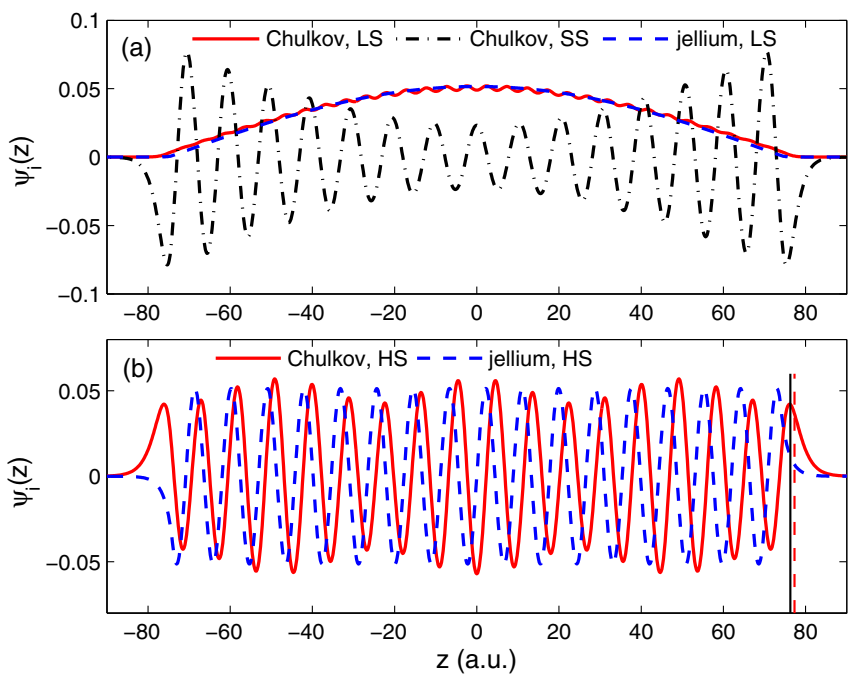

FIG. 1. (Color online) Valence-band initial-state wave functions of a 31-layer $\mathrm{Mg}(0001)$ slab. (a) The lowest valence state (LS; red solid line for the Chulkov potential and blue dashed line for the jellium potential) and the lower surface state (SS; black dash-dotted line for the Chulkov potential). (b) The highest valence state (HS; red solid line for the Chulkov potential and blue dashed line for the jellium potential).

calculations for the $\operatorname{Mg}(0001)$ surface yields the four independent parameters $A_{10}=-10.55 \mathrm{eV}, A_{1}=0.70 \mathrm{eV}, A_{2}=$ $1.26 \mathrm{eV}$, and $\beta^{\prime}=4.7223$ [36]. The remaining parameters in $U_{C}(z)$ are obtained by imposing the continuity of the logarithmic derivative of the potential for all values of $z$.

In particular, the image-plane position for the $\operatorname{Mg}(0001)$ surface follows from (17) and the four independent parameters given above as $z_{i m}=\left(d-a_{s}\right) / 2+3.46$ [21]. Since the metalvacuum interface position in the Chulkov model is not well defined due to the decrease of (17) toward the vacuum, we choose it to be identical with the image plane position and set $\left|z_{i}\right|=z_{i m}$. In calculations with Chulkov initial states, we therefore assume the onsets of the Volkov final-state damping in (4) and screening for the IR laser electric field in (12) to coincide with the image-plane position. This choice of placing the metal-vacuum interface position for Chulkov-model calculations outside the interface position we

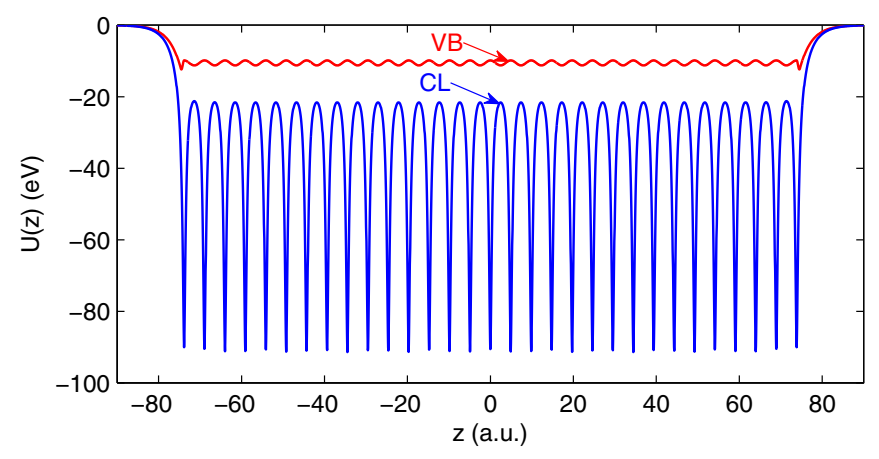

FIG. 2. (Color online) Effective model potentials $U_{C}(z)$ and $U_{C}(z)+U_{Y}(z)$ for the valence electronic structure (top red line) and $2 p$ core-level band (lower blue line), respectively, for a 31-layer $\operatorname{Mg}(0001)$ slab. use for jellium initial states $\left(\left|z_{i}\right|=d / 2\right)$ is consistent with the probability density of the highest occupied VB state for the Chulkov potential extending over a slightly larger spatial range than the probability density of the highest occupied jellium state [Fig. 1(b)].

Diagonalizing the corresponding Hamiltonian, $-\partial^{2} /\left(2 \partial z^{2}\right)+U_{C}(z)$, yields 35 occupied projected VB states below the Fermi level. All VB states are highly delocalized (Fig. 1). Relating electronic energies to the Fermi level $\varepsilon_{F}$, we find the lowest and highest occupied states at $\epsilon_{\mathrm{LS}}=-6.90 \mathrm{eV}$ and $\epsilon_{\mathrm{HS}}=-0.10 \mathrm{eV}$, respectively. For the 31-layer $\mathrm{Mg}$ thin film we thus calculated the projected $\mathrm{VB}$ width according to $\epsilon_{\mathrm{HS}}-\epsilon_{\mathrm{LS}}=6.80 \mathrm{eV}$. This value exceeds the band width measured for $\mathrm{Mg}(0001)$ single crystals $(6.15 \mathrm{eV})$ [37]. Two surface states with energies $\epsilon_{\mathrm{SS}}^{1}=-1.53 \mathrm{eV}$ and $\epsilon_{\mathrm{SS}}^{2}=-1.46 \mathrm{eV}$ are located inside the $\sim 1.1 \mathrm{eV}$-wide band gap covering the interval between $-1.94 \mathrm{eV}$ and $-0.8 \mathrm{eV}$. Our values for the two surface-state energies are close to the measured value $(\sim-1.6 \mathrm{eV})$ [37]. The wave function of the energetically lower surface state is shown in Fig. 1(a), together with the wave functions for the lowest VB state within both the Chulkov and jellium model. The wave functions of the highest occupied VB state in either model are shown in Fig. 1(b).

\section{Emission from core-level states}

\section{Zero-bandwidth model}

The localized CL states can be modeled by zero-bandwidth tight-binding initial wave functions [18,25]. These wave functions are constructed by superimposing identical isolated $2 p$ atomic wave functions $\phi_{2 p}$ with binding energy $\varepsilon_{i}=\varepsilon_{2 p}$ that are centered at the lattice points $\left\{z_{j}\right\}$ of the substrate,

$$
\psi_{i}(z)=\sum_{j}\left\{e^{i k_{i, z}^{(n)}(z-d / 2)}-e^{-i k_{i, z}^{(n)}(z-d / 2)}\right\} \phi_{2 p}\left(z-z_{j}\right),
$$

where $k_{i, z}^{(n)}=n \pi / d\left(n=1, \ldots, d / a_{s}\right)$ and $z_{j}=d-(j-1 / 2) a_{s}$ $\left(j=1, \ldots, d / a_{s}\right)$. The stationary initial state $\psi_{i}(z)$ is composed of incident and reflected Bloch waves with the crystal momenta $\pm k_{i, z}^{(n)}$ that describe the motion of an electron inside the solid towards and away from the metal-vacuum interface. The metal-vacuum interface positions for the zerobandwidth model are at $\left|z_{i}\right|=d / 2$. The atomic wave functions $\phi_{2 p}(z)$ are modeled as the ground state of the soft-core Coulomb potential $V(z)=-1 / \sqrt{\left(z^{2}+a^{2}\right)}$, and the softcore parameter $a=0.28$ is adjusted to yield the measured central binding energy of the magnesium $2 p \mathrm{CL}$ band, $49.6 \mathrm{eV}$ (relative to $\epsilon_{F}$ ) [35].

\section{Chulkov-Yukawa model}

In the Chulkov-Yukawa model $[17,22,28]$, the effective potential for the $2 p$ CL states is modeled by combining the Yukawa potential,

$$
U_{Y}(z)=-\sum_{j} e^{-\left|z-z_{j}\right| / \xi} / \sqrt{\left(z-z_{j}\right)^{2}+a_{Y}^{2}},
$$

with the Chulkov potential (16). The effective CL potential $U_{\mathrm{CL}}=U_{C}(z)+U_{Y}(z)$ is shown as the lower blue line in 


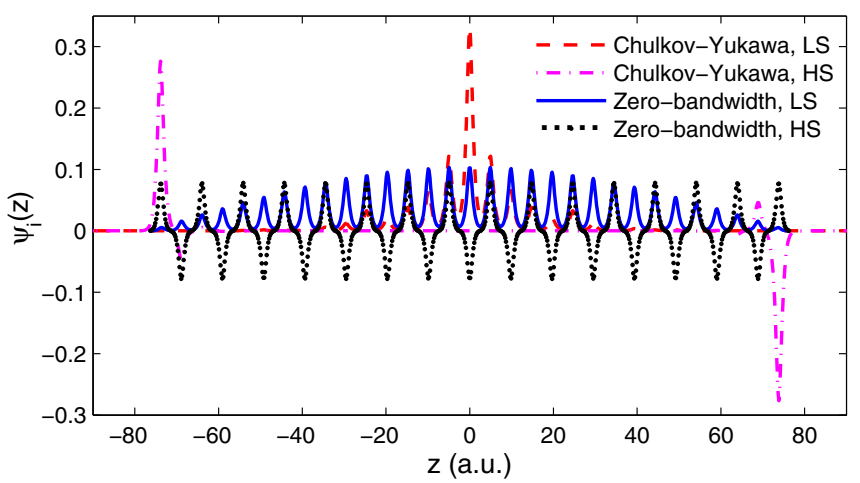

FIG. 3. (Color online) Lowest $2 p$ core-level wave functions (LS; red dashed line for the Chulkov-Yukawa potential, blue solid line for the zero-bandwidth potential) and the highest $2 p$ core-level wave functions (HS; magenta dash-dotted lines for the Chulkov-Yukawa potential, black dotted line for the zero-bandwidth potential) for a 31-layer $\operatorname{Mg}(0001)$ slab.

Fig. 2. The screening length $\xi$ and soft-core parameter $a_{Y}$ control the CL binding energies and bandwidth. Adjusting $\xi=3$ and $a_{Y}=0.344$ yields the $\operatorname{Mg}(2 p)$ CL states with measured binding energies (centered near $-50 \mathrm{eV}$ ) and a narrow bandwidth of $0.9 \mathrm{eV}$, close to the measured bandwidth of $\sim 0.6 \mathrm{eV}$ [4].

The energetically highest $2 p$ CL wave functions have enhanced amplitudes at the slab surfaces (Fig. 3). This is consistent with the fact that the $\mathrm{Mg} 2 p$ CL states have distinct surface and bulk contributions that can be distinguished in measured high-resolution synchrotron $2 p$ CL PE spectra [4]. In contrast, the corresponding zero-bandwidth-model wave functions do not have an enhanced amplitude at the surfaces of the thin film. The energetically lowest $2 p \mathrm{CL}$ wave function is strongly enhanced at the slab center, while the zero-bandwidth model predicts a smoothly varying amplitude of the corresponding lowest wave function.

\section{NUMERICAL RESULTS}

\section{A. Emission from valence-band states}

\section{Jellium initial states}

VB streaking spectrograms calculated within the jellium model in general oscillate with the NIR vector potential $A_{L}(t)$ as shown in Figs. 4(a)-4(j), 4(n), and 4(o), except for calculations with infinite MFP and finite skin depth [Figs. 4(k)-4(m)]. As stated earlier, all PE kinetic energies are given relative to $\epsilon_{F}$. The detailed energy- and delay-dependent structure of the streaking traces, i.e., the delay-dependent dispersion of the PE wave packet [8], depends on the MFP $\lambda$, XUV-chirp rate $\beta$, and NIR skin depth $\delta_{L}$.

The value of the skin depth in metals for short intense NIR pulses is a matter of ongoing debate. Different recent numerical simulations have reproduced the measured relative streaking delay between $\mathrm{VB}$ and $\mathrm{CL}$ photoemission from the W(110) surface [3], employing NIR skin depths of $2 \AA$ [17], $100 \AA$ [18], and $\sim 400 \AA$ [16]. In a previous quantum-mechanical simulation [22], we used a NIR skin depth of $2 \AA$ and the NIR-pulse phase $\varphi=1.22 \pi$ in (14) to best reproduce the measured [4] streaked PE spectra from a $\operatorname{Mg}(0001)$ surface. We therefore first consider the skin depth $\delta_{L}=2 \AA$ and assume $\varphi=1.22 \pi$ throughout this work. For XUV pulses without chirp $(\beta=0)$ and for $\lambda$ increasing from 0.5 to $5 \AA$, the COEs of the streaking traces sightly shift towards lower kinetic energies [cf., e.g., Figs. 4(b) and $4(\mathrm{~g})]$. This is because the electronic probability density of individual jellium initial states with lower energies increases with increasing depth. Their contribution to the total yield thus becomes larger as the MFP increases. The PE spectral width is XUV-NIR-pulse-delay dependent for chirped XUV pulses, and the streaking trace patterns are asymmetric within the delay range of one NIR optical cycle. For negative chirp [Figs. 4(a), 4(d), 4(f), and 4(i)], the peak intensity of the PE spectrum is reduced in the "up-streaking" delay ranges (defined as with $\tau$ increasing final PE kinetic energies), and is enhanced in adjacent "down-streaking" delay ranges (defined as with increasing $\tau$ decreasing final PE kinetic energies). This asymmetry is reversed for positive chirp values [Figs. 4(c) and $4(\mathrm{~h})$ ].

The streaking-delay-dependent change of the PE spectral profile is more pronounced for $\lambda=0.5 \AA$ than for $\lambda=5 \AA$ (Fig. 4), irrespective of the chirp of the XUV pulse. This increase of delay-dependent spectral changes with decreasing MFP is due to dominant contributions from the energetically highest occupied VB states to the PE spectrum for $\lambda \ll a_{s}$, promoting comparatively narrow spectral profiles. In contrast, for $\lambda>a_{s}$, PEs from lower states significantly contribute to the VB PE spectrum, leading to broadened spectral profiles.

For finite NIR skin depth $\left(\delta_{L}=2 \AA\right)$ and infinite MFP, the streaking amplitudes in Figs. $4(\mathrm{k})-4(\mathrm{~m})$ become very small and no longer map the oscillatory character of the NIR electric field. This effect can be traced to the Volkov phase (8): for finite NIR skin depths, the action of the streaking IR laser field is limited to a very small spatial interval on the scale of the NIR wavelength. In addition, for an infinite MFP, electrons that are released within a large spatial range arrive close to the surface where they get exposed to the NIR field. They have thus accumulated different phases that, on average, smear out the streaking oscillations, resulting in nonoscillatory streaking traces. In contrast, for $\delta_{L}=\infty$ and infinite MFP, the familiar oscillatory behavior of streaking energy shifts reappears [Figs. 4(n) and 4(o)]. In this case PEs are streaked immediately following their release upon absorption of an XUV photon, irrespective of the location of their release. Therefore, all PEs accumulate comparable phases relative to the phase of the streaking laser electric field and imprint the same oscillatory delay dependence on the PE yield.

\section{Chulkov initial states}

Streaked VB spectra calculated with Chulkov-model initial states for a 31-layer $\operatorname{Mg}(0001)$ slab are shown in Fig. 5. Overall, these photoemission spectra are very similar to those obtained with jellium-model initial states in Fig. 4. As for the jellium model, the delay-dependent asymmetry of the photoemission yield is reversed by changing the sign of the XUV chirp parameter, and the spectral peak intensity of the photoemission spectra is reduced in the up-streaking delay ranges for negative XUV chirp. For both initial state 


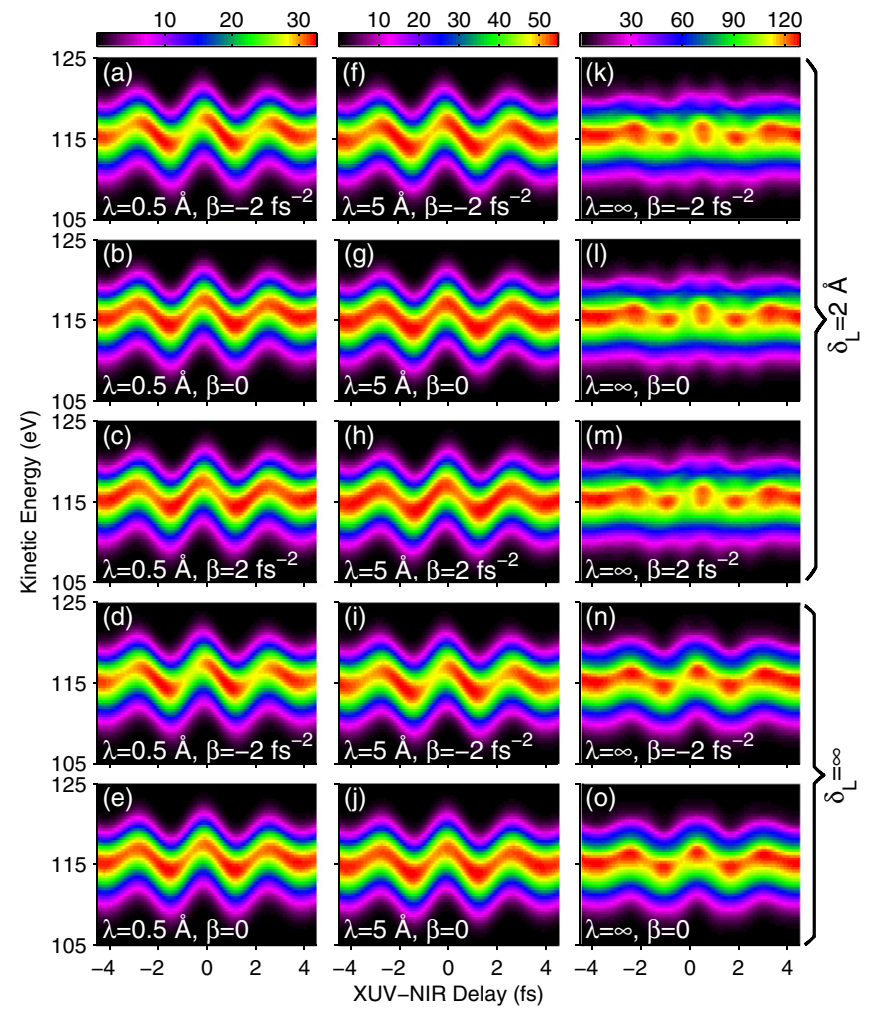

FIG. 4. (Color online) Valence-band streaking spectra calculated with jellium initial states for a 31-layer $\operatorname{Mg}(0001)$ slab and different mean free paths $\lambda$, XUV-pulse chirp rates $\beta$, and streaking-field skin depths $\delta_{L}$ : (a)-(e) $\lambda=0.5 \AA$, (f)-(j) $\lambda=5 \AA$, and (k)-(o) $\lambda=\infty$; (a),(d),(f),(i),(k),(n) $\beta=-2 \mathrm{fs}^{-2},(\mathrm{~b}),(\mathrm{e}),(\mathrm{g}),(\mathrm{j}),(\mathrm{l}),(\mathrm{o}) \beta=0$, and (c),(h),(m) $\beta=2 \mathrm{fs}^{-2}$; (a)-(c), (f)-(h), (k)-(m) $\delta_{L}=2 \AA$, and (d),(e),(i),(j),(n),(o) $\delta_{L}=\infty$.

models, the $\tau$-dependent change of the PE spectral profile for negative XUV chirp is a little stronger than for positive chirp. The XUV-chirp-dependent spectral-amplitude change is better seen for the fixed XUV-NIR delay, $\tau=-0.79$ fs, in Fig. 6, for both jellium [Fig. 6(a)] and Chulkov initial states [Fig. 6(b)].

Despite these similarities, closer inspection reveals that the peak intensities of PE spectra for fixed XUV-NIR delays appear at different energies within the Chulkov and jellium models (Fig. 6). As the MFP decreases from $5.0 \AA$ to $0.5 \AA$, the peak energy as a function of the XUV chirp rate shifts by a larger amount, with a larger increase in peak yield, for the Chulkov model. These dependencies for the two initial-state models imply that band-structure effects influence streaked PE spectra. In turn, the comparison of measured and calculated streaked PE spectra might be used to scrutinize electronic band-structure models.

For infinite NIR skin depth and infinite MFP, both initialstate models predict $\mathrm{COE}$ shifts that oscillate in phase with the negative vector potential $\left(-A_{L}\right)$ of the streaking laser pulse, as shown in Fig. 7. For infinite NIR skin depth (in practice, for skin depths that are much larger than the electron MFP) nonzero $\mathrm{VB}$ photoemission streaking time delays relative to $-A_{L}$ are thus due to finite MFPs [18] and appear to necessitate the scattering of PEs during their propagation inside the solid. Interestingly, for finite electron MFPs, the VB photoemission delay is only weakly dependent on both MFP and the screening

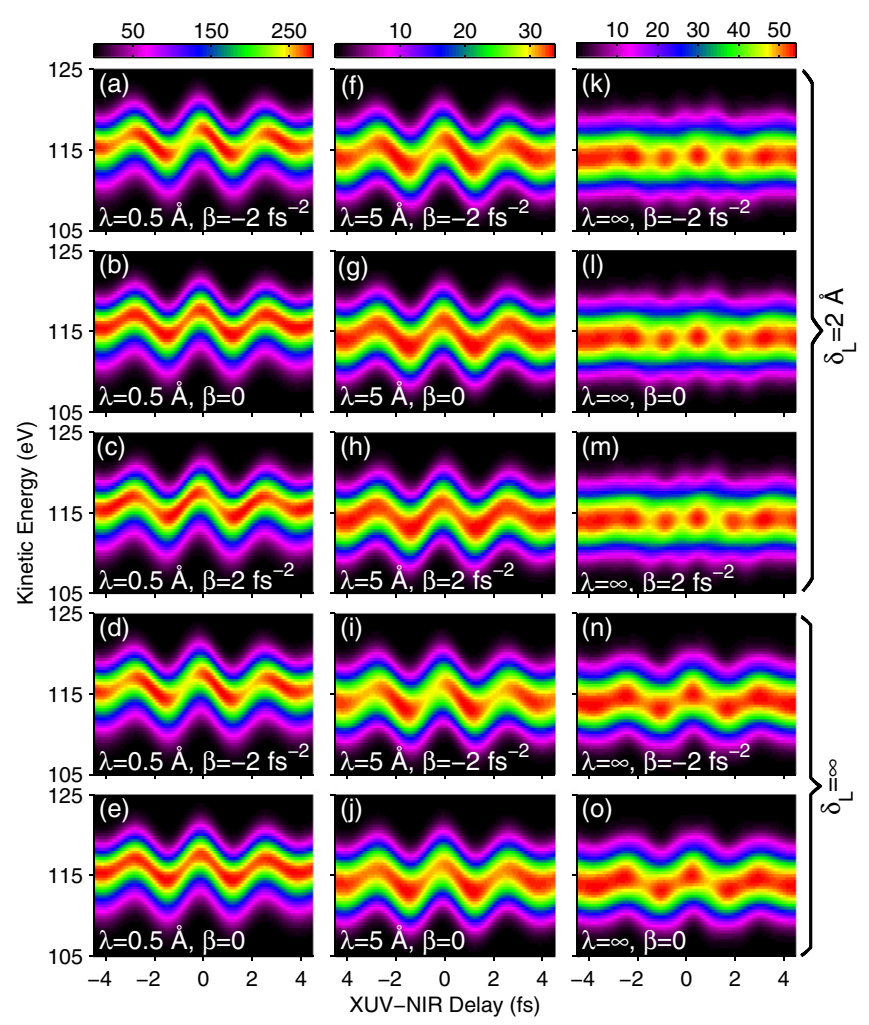

FIG. 5. (Color online) Same as Fig. 4 but for Chulkov initial states.

of the streaking field inside the solid (Fig. 8). For MFPs $\lambda$ between 0 and $8 \AA$ and NIR skin depth $\delta_{L}$ between $2 \AA$ and $\infty$, both initial-state models predict photoemission delay changes of less than 12 as. For jellium initial states, the VB photoemission delay is independent of the electron MFP and NIR skin depth for $1 \AA \leqslant \lambda \leqslant 8 \AA$. In contrast, for Chulkov initial states, the magnitude of the photoemission delay gradually increases by 10 as, as $\lambda$ increases from 0.1

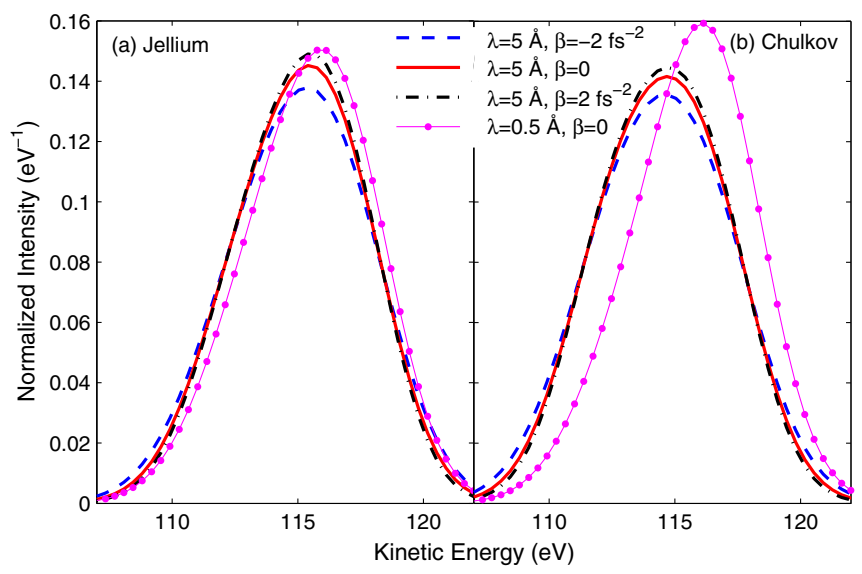

FIG. 6. (Color online) Valence-band photoemission spectra for three different XUV chirp rates $\beta$, NIR skin depth $\delta_{L}=2 \AA$, and two different mean free paths $\lambda$. These spectra show the data in (a) Figs. 4(b), 4(f)-4(h) and (b) Figs. 5(b), 5(f)-5(h) for the fixed XUV-NIR pulse delay $\tau=-0.79 \mathrm{fs}$. 


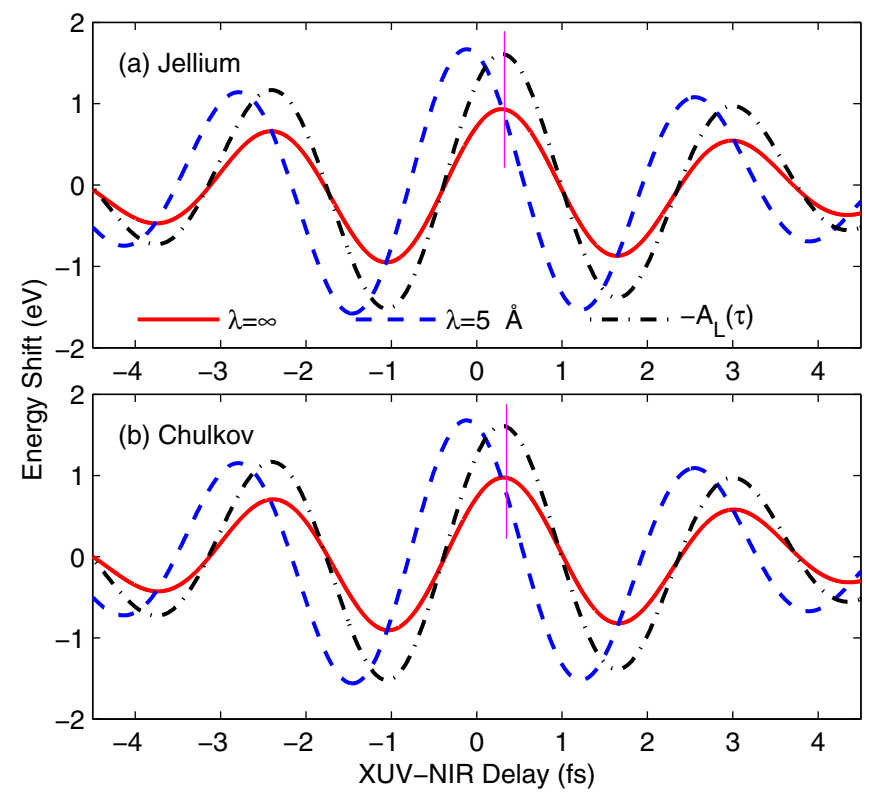

FIG. 7. (Color online) Center-of-energy shifts for streaked XUV photoemission from the valence band of a 31-layer $\operatorname{Mg}(0001)$ slab as a function of XUV-NIR pulse delay, obtained by spectrally averaging the spectrograms in (a) Figs. 4(i) and 4(n) and (b) Figs. 5(i) and 5(n) for $\delta_{L}=\infty$ and $\beta=-2 \mathrm{fs}^{-2}$. As a reference, the vector potential $-A_{L}(\tau)$ is shown in arbitrary units in both graphs. The vertical lines show the peak positions of the COE shifts for $\lambda=\infty$ and of $-A_{L}(\tau)$.

to $5 \AA$. It continues to increase at a much slower rate as $\lambda$ increases beyond $5 \AA$.

For screened NIR streaking fields and skin depth $\delta_{L}=2 \AA$, the photoemission delay changes by less than 7 as for $1 \AA \leqslant \lambda \leqslant 8 \AA$ (Fig. 8). However, this change and the VB photoemission time delay depend on the position at which the onset of the NIR-field screening is assumed. Therefore, while

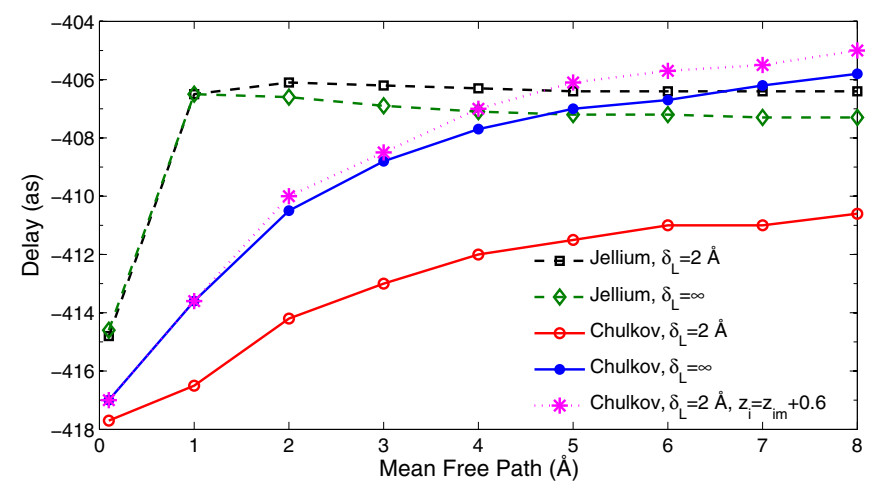

FIG. 8. (Color online) Photoemission time delays for streaked XUV electron emission from the valence band of a 31-layer $\operatorname{Mg}(0001)$ slab by a chirped XUV pulse with $\beta=-2 \mathrm{fs}^{-2}$. The streaked photoemission delay for jellium and Chulkov initial states is calculated relative to the phase of the negative NIR pulse vector potential, $-A_{L}(\tau)$, and shown as a function of the electron mean free path for different skin depths $\delta_{L}$ of the streaking field. The magenta asterisks and dotted line indicate the photoemission delays calculated for $\delta_{L}=2 \AA$ by increasing $z_{i}=z_{i m}$ to $z_{i}=z_{i m}+0.6$ for Chulkov initial states. comparing photoemission time delays derived from Chulkov and jellium initial states, one needs to keep in mind that the jellium electron probability density decays faster with the distance from the slab than the probability density of Chulkov initial states, as shown in Fig. 1(b). For consistency, this suggests that the onset of NIR-field screening for Chulkov initial states should be taken at a slightly larger distance from the topmost lattice plane of the substrate than for jellium initial states. By shifting the interface position by 0.6 to $z_{i}=z_{\text {im }}+0.6$, the magnitude of the VB photoemission time delays calculated with Chulkov initial states increases by $\approx 6$ as (magenta asterisks and dotted line in Fig. 8). This increase is consistent with the classical propagation time of $\approx 5$ as needed by the PE to travel a distance of 0.6 with a kinetic energy of $115 \mathrm{eV}$. It also moves streaking delays calculated within the Chulkov model closer to the delays obtained within the jellium model for $\lambda=5 \AA$.

\section{B. Emission from core-level states}

\section{Zero-bandwidth initial states}

Figure 9 shows streaked photoemission spectra, calculated by modeling the $2 p$ initial states within the zero-bandwidth model for different MFPs, XUV-chirp rates, and NIR skin depths. Overall, the $2 p$ streaking traces are similar to the VB streaking traces in Fig. 4. As for VB photoemission, for $\lambda=\infty$ and $\delta_{L}=2 \AA$, we do not obtain the familiar oscillating streaking traces. However, the spectral shape of the $2 p$ streaking pattern in Fig. 9 is even less dependent on the MFP for finite values of $\lambda$, and the $\tau$-dependent $2 p$ PE spectral profile is more sensitive to the XUV chirp than the VB PE spectral profiles in Fig. 4. Comparing the streaking patterns in Fig. 9 for $\delta_{L}=2$ with those for $\delta_{L}=\infty$, we find that the screening of the streaking field modifies the XUV-NIR-pulse-delay dependent change of the $2 p \mathrm{PE}$ dispersion, most significantly for $\lambda=\infty$.

\section{Chulkov-Yukawa initial states}

Figure 10 shows streaked photoemission spectra, calculated by modeling the $2 p$ initial states within the Chulkov-Yukawa model for different MFPs, XUV-chirp rates, and NIR skin depths. We find no major differences between the photoemission profiles obtained within the zero-bandwidth and ChulkovYukawa models in Figs. 9 and 10, respectively. However, the PE yields calculated with zero-bandwidth initial state for $\lambda=1 \AA$ exceed those obtained with Chulkov-Yukawa initial states. This is compatible with the dominantly contributing energy initial states in the zero-bandwidth model having larger probability density at the top layers of the magnesium film than for the Chulkov-Yukawa potential (cf. Fig. 3).

A detailed quantitative comparison of $2 p$ PE spectra obtained for the two initial-state models is presented in Fig. 11 for the fixed XUV-NIR pulse delay $\tau=-0.79$ fs and $\lambda=4 \AA$. All PE spectra map the Gaussian temporal profile of the XUV pulse. The large difference in the electronic probability density distribution of CL states modeled in zero-bandwidth and Chulkov-Yukawa approximation shown in Fig. 3 does not result in easily distinguishable differences in the PE spectral profiles. This implies that the spectral profile of the $2 p$ PE wave 


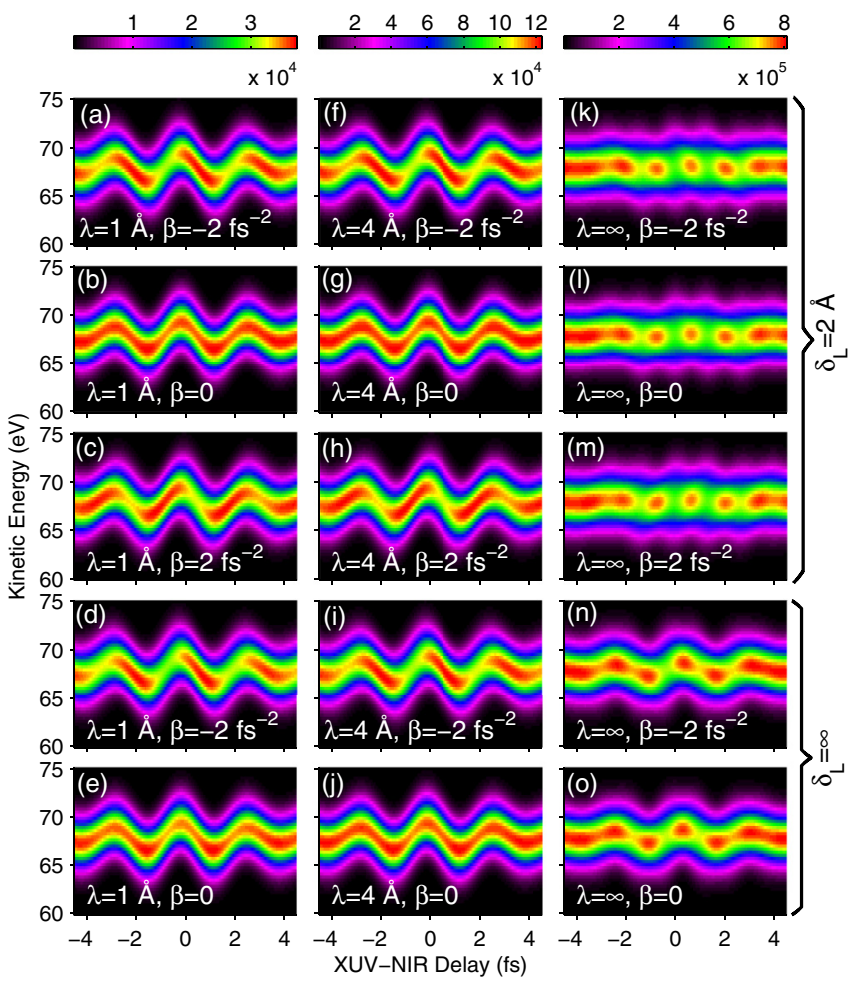

FIG. 9. (Color online) Streaked $2 p$ photoemission spectra, calculated by modeling the initial core-level states within the zerobandwidth model for a 31-layer $\operatorname{Mg}(0001)$ slab and different mean free paths $\lambda$, XUV-pulse chirp rates $\beta$, and streaking-field skin depths $\delta_{L}$ : (a)-(e) $\lambda=1 \AA$, (f)-(j) $\lambda=4 \AA$, and (k)-(o) $\lambda=\infty$; (a),(d),(f),(i),(k),(n) $\beta=-2 \mathrm{fs}^{-2}$,(b),(e),(g),(j),(l),(o) $\beta=0$, and (c),(h),(m) $\beta=2 \mathrm{fs}^{-2}$; (a)-(c),(f)-(h),(k)-(m) $\delta_{L}=2 \AA$ and (d),(e),(i),(j),(n),(o) $\delta_{L}=\infty$.

packet is determined by the XUV pulse and the screening of the NIR streaking field. The dominant transfer of the XUV-pulse spectral profile to the PE wave packet for $2 p \mathrm{CL}$ emission is consistent with the spectral profiles for CL emission in Fig. 11 depending more sensitively on the XUV chirp than the profiles shown for VB emission in Fig. 6.

As for VB emission (cf. Fig. 7), for infinite NIR skin depth and infinite MFP, both $2 p$ initial-state models predict COE shifts of the streaking traces that oscillate in phase with the negative vector potential $\left(-A_{L}\right)$ of the streaking laser pulse (Fig. 12). As discussed at the end of Sec. III A 1 above for VB emission, we again confirm for $2 p$ CL emission that for infinite NIR skin depth nonzero photoemission streaking time delays relative to $-A_{L}$ require finite MFPs, i.e., the scattering of released $2 p$ PEs during their propagation inside the solid.

The dependence of the $2 p$ photoemission delay [relative to the phase of the streaking field $\left.\left(-A_{L}\right)\right]$ on the electron MFP and the NIR skin depth of the streaking field is shown in Fig. 13. Compared to VB photoemission (cf. Fig. 8), for efficient screening of the streaking field $\left(\delta_{L}=2 \AA\right)$, the $2 p$ photoemission time delay more significantly changes with the electron MFP. For $2 p$ CL emission, the photoemission delay increases by $\approx 50$ as within the zero bandwidth and $\approx 65$ as within the Chulkov-Yukawa model as $\lambda$ increases from $1 \AA$ to $8 \AA$. In contrast, for an unscreened NIR streaking

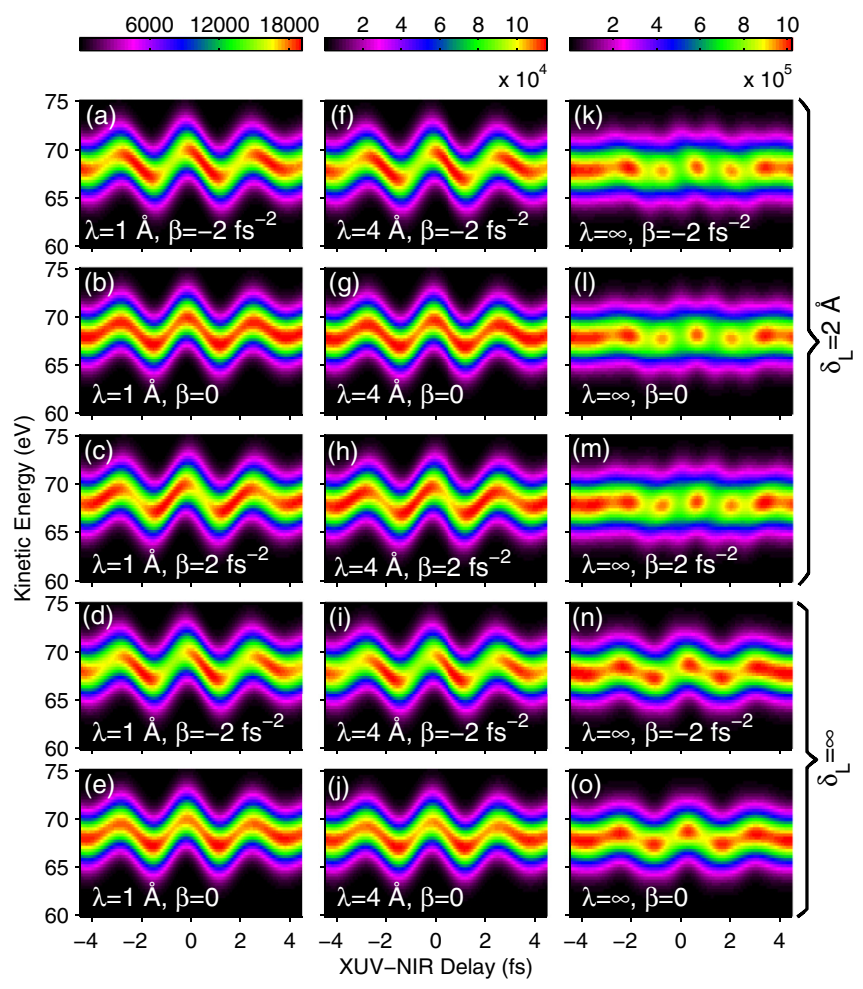

FIG. 10. (Color online) Same as Fig. 9 but for Chulkov-Yukawa initial states.

field $\left(\delta_{L}=\infty\right)$, the photoemission delay increases by only $\approx 10$ as for both initial-state models over the same MFP interval. The magnitude of the photoemission time delay for Chulkov-Yukawa initial states is smaller than the magnitude of the delay obtained with the zero-bandwidth model for the same MFP and skin depth values, especially for finite skin depth. The significant difference in the photoemission delay between the two $2 p$ initial-state models is due to the significantly different probability density distributions of their initial-state wave functions (cf. Fig. 3).

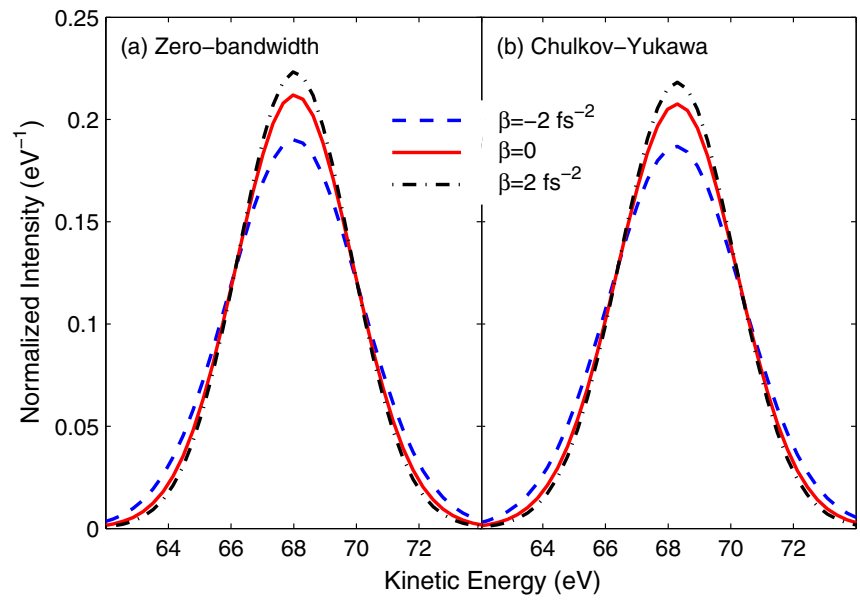

FIG. 11. (Color online) $2 p$ photoemission spectra for the mean free path $\lambda=4 \AA$, NIR skin depth $\delta_{L}=2 \AA$, and three different XUV chirp rates $\beta$, showing the data in (a) Figs. 9(f) -9 (h) and (b) Figs. 10(f)-10(h) for the fixed XUV-NIR pulse delay $\tau=-0.79$ fs. 

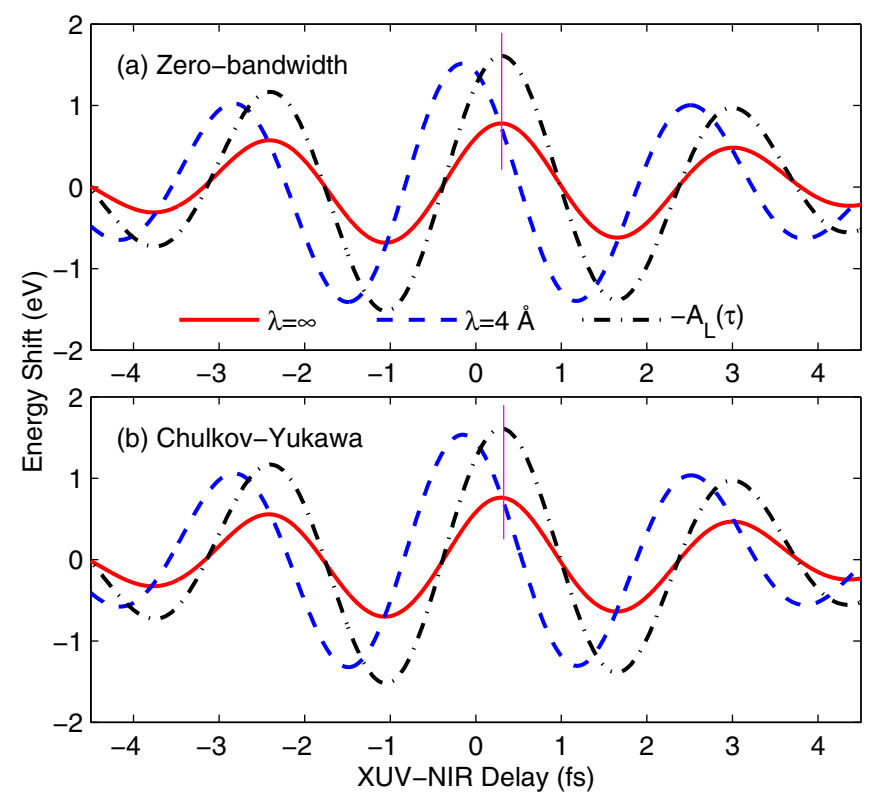

FIG. 12. (Color online) Center-of-energy shifts for streaked XUV photoemission from the $2 p$ core-level band of a 31-layer $\operatorname{Mg}(0001)$ slab as a function of XUV-NIR pulse delay, obtained by spectrally averaging the spectrograms in (a) Figs. 9(i) and 9(n) and (b) Figs. 10(i) and $10(\mathrm{n})$ for $\delta_{L}=\infty$ and $\beta=-2 \mathrm{fs}^{-2}$. As a reference, the vector potential $-A_{L}(\tau)$ is shown in arbitrary units in both graphs. The vertical lines show the peak positions of the COE shifts for $\lambda=\infty$ and of $-A_{L}(\tau)$.

The magnitude of the $2 p$ photoemission time delays shown in Fig. 13 increases linearly with the electron MFP for both initial-state models. Moreover, the difference in our calculated photoemission delays between $\delta_{L}=2 \AA$ and $\infty$ is in qualitative agreement with the variation as a function of $\delta_{L}$ of the classical photoemission delay. The classically estimated

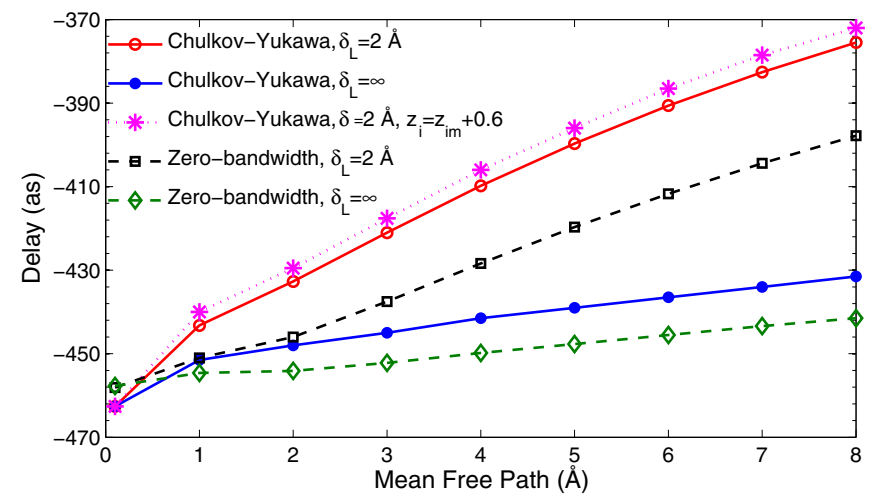

FIG. 13. (Color online) Photoemission time delays for the NIRstreaked XUV electron emission from the $2 p$ core-level band of a 31-layer $\operatorname{Mg}(0001)$ slab by chirped XUV pulses with $\beta=-2 \mathrm{fs}^{-2}$. The streaked photoemission delays for zero-bandwidth and ChulkovYukawa initial states are given relative to the phase of the negative NIR pulse vector potential, $-A_{L}(\tau)$, and shown as a function of the electron mean free path for different skin depths $\delta_{L}$ of the streaking field. The magenta asterisks and dotted line indicate the photoemission delays calculated for $\delta_{L}=2 \AA$ and Chulkov-Yukawa initial states after increasing $z_{i}$ by 0.6 to $z_{i}=z_{i m}+0.6$. photoemission delay is given as the average traveling time, $\left(\lambda-\delta_{L}\right) / v_{e}$, a PE needs to move from its release location at $z=z_{i}-\lambda$ to the onset of the streaking field at $z=z_{i}-\delta_{L}$ at a constant speed $v_{e}$. For example, a $2 p$ PE released at the distance $\lambda=4 \AA$ below the interface position travels for about 40 as before entering the streaking field with $\delta_{L}=2 \AA$. This classical travel time agrees with the difference of our quantummechanically calculated results between $\delta_{L}=2 \AA$ and $\infty$ for Chulkov-Yukawa initial states (Fig. 13). Similarly, for $\lambda=6 \AA$, the quantum-mechanical prediction of the $2 p$ photoemission delay is $\sim 60$ as, close to the classical estimation of $\sim 80$ as.

Figure 14(a) shows the dependence of the $2 p$ photoemission delay predicted by the two initial-state models on the skin depth of the streaking field for $\lambda=4 \AA$. The change of the photoemission delay between $\delta_{L}=2 \AA$ and $\infty$ predicted by the zero-bandwidth model is 10 as less than that predicted within the Chulkov-Yukawa model. The dependence of the photoemission delay on the skin depth is stronger for smaller values of the skin depth. Within the Chulkov-Yukawa model, we find that the photoemission delay decreases by $\approx 40$ as for $\delta_{L}$ increasing from 0 to $6 \AA$. As $\delta_{L}$ further increases to $\infty$, the delay continues to decrease by 20 as. The difference in photoemission delays between the two initial-state models reduces from 20 as to 8 as as we increase the NIR skin depth from 0 to $\infty$.
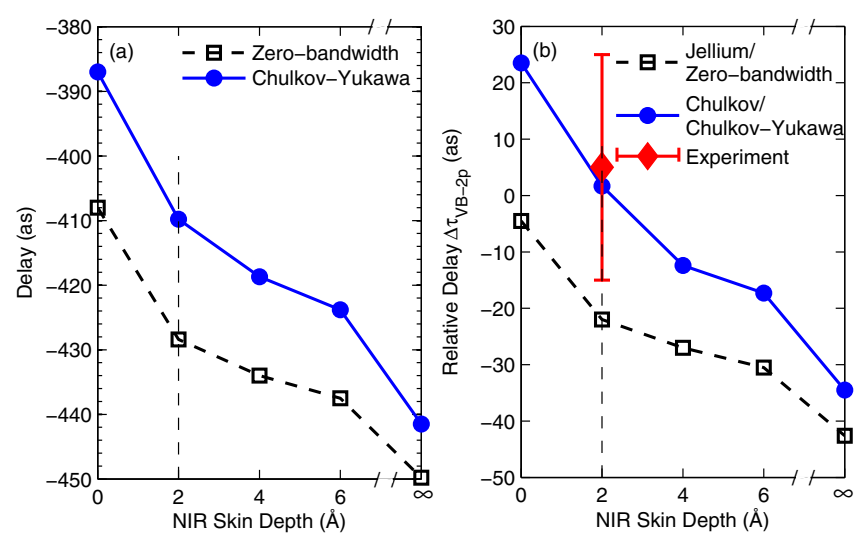

FIG. 14. (Color online) (a) Photoemission time delays [relative to $-A_{L}(\tau)$ ] for the NIR-streaked XUV electron emission from the $2 p$ core-level band of a 31-layer $\operatorname{Mg}(0001)$ slab. The streaked photoemission delays for zero-bandwidth (black squares and dashed line) and Chulkov-Yukawa (blue dots and solid line) initial states are given relative to the phase of the negative NIR pulse vector potential, $-A_{L}(\tau)$ and shown as a function of skin depth $\delta_{L}$ of the NIR streaking field for the electron mean free path $\lambda=4 \AA$. (b) Relative photoemission streaking time delay $\Delta \tau_{V B-2 p}$ obtained as the difference between the VB photoemission delay, calculated with Chulkov initial states, and the $2 p$ core-level delay, calculated with Chulkov-Yukawa initial states, as a function of the skin depth $\delta_{L}$ (blue dots and solid line). Black squares interpolated with dashed lines show $\Delta \tau_{V B-2 p}$ calculated based on jellium VB and $2 p$ zero-bandwidth initial states. The mean free paths for valence-band and core-level emission in this calculation are $5 \AA$ and $4 \AA$, respectively. The red diamond with error bar shows the experimental results of Neppl et al. [4]. (a), (b) The XUV-chirp parameter is $-2 \mathrm{fs}^{-2}$. The vertical dashed lines indicate the assumed value for the skin depth that best reproduces the experiment. 


\section{Relative photoemission streaking time delays for valence-band versus core-level emission}

As for VB photoemission, streaked CL photoemission time delays depend on the position at which the NIR-field screening is assumed to start. If we shift the interface position from $z_{i}=z_{\text {im }}$ to $z_{i}=z_{\text {im }}+0.6$, the photoemission time delay for Chulkov-Yukawa initial states changes by $\approx 4$ as (magenta asterisks and dotted line in Fig. 13), in qualitative agreement with the classical estimate of 6.5 as needed by a PE to travel a distance of 0.6 at a kinetic energy of $68 \mathrm{eV}$. The shift of $\approx 4$ as is comparable with the shift of $\approx 6$ as we found for VB photoemission for the same change of the onset of NIR screening (cf. Fig. 8). From this study, we estimate that the not well-defined onset of NIR screening at the surface of the magnesium slab induces an uncertainty of $\approx 2$ as in the relative photoemission delay $\Delta \tau_{V B-2 p}$. This uncertainty lies well within the experimental error, \pm 20 as, for $\Delta \tau_{V B-2 p}$ in the experiment of Neppl et al. [4], so that more accurate estimates for the onset of NIR screening cannot be deduced from the available experimental data.

Since the VB photoemission time delays for both jellium and Chulkov initial states are comparatively insensitive to variations of the streaking-field skin depth, the dependence of the relative delay $\Delta \tau_{V B-2 p}$ on the streaking-field skin depth is decided by the sensitivity of $2 p$ CL photoemission to the skin depth as shown in Fig. 14(b). The difference between the relative delay $\Delta \tau_{V B-2 p}$ based on Chulkov VB and $2 p$ Chulkov-Yukawa initial states and that based on jellium VB and $2 p$ zero-bandwidth initial states, increases from 7 as to 28 as, as the skin depth decreases from $\infty$ to 0 . For a skin depth of $2 \AA$, the jellium VB and $2 p$ zero-bandwidth initial states give rise to a relative delay $\Delta \tau_{V B-2 p}$ that exceeds in magnitude the error range of the experimental delay [4].

\section{CONCLUSIONS}

In summary, we have systematically investigated the dependence of NIR-streaked XUV photoemission spectrograms and time delays for photoemission from a $\mathrm{Mg}(0001)$ surface on the modeling of the initial VB and CL electronic states, the electron MFP, and the skin depth of the NIR streaking field. For delocalized VB states, the XUV-NIR delay-dependent dispersion of the PE spectral profiles, as mapped in streaked PE spectra, is sensitive to the XUV chirp and depends on the electronic band structure, electron MFP, and the screening of the NIR streaking field.
The streaked photoemission time delay for emission from the VB is found to be less sensitive to details of the $\operatorname{Mg}(0001)$ electronic band structure, electron MFP, and the screening of the NIR streaking field than the delay for CL emission (Figs. 8 and 13). For photoemission from the localized CL band states, the XUV-NIR delay-dependent dispersion of the $\mathrm{PE}$ spectral profile is more sensitive to the XUV chirp than for VB emission due to the narrow bandwidth of the $2 p$ band (Figs. 6 and 11). We found no significant differences in the VB PE spectral profiles for jellium and Chulkov initial states (Figs. 4 and 5). Similarly, the CL PE spectral profiles calculated with zero-bandwidth and Chulkov-Yukawa initial states are very similar (Figs. 9 and 10).

For different initial-state models, the large discrepancy between electronic probability density distributions gives rise to significant variations in the photoemission time delay difference between $\mathrm{CL}$ and VB emission. The vanishing relative time delay between $\mathrm{VB}$ and $2 p \mathrm{CL}$ photoemission from a $\operatorname{Mg}(0001)$ surface in the experiment of Neppl et al. [4] is reproduced (within the experimental error bar) only with the more realistic model potentials (Chulkov VB and $2 p$ ChulkovYukawa initial states) in the quantum-mechanical simulations discussed in this work. While this tends to validate our use of these initial-state models, we need to point out, however, that the relative streaking delays between photoemission from VB and CL states also depend on different (energy-dependent) MFPs and the efficient screening of the streaking field at the surface.

The values we adopted for the MFPs (5 $\AA$ and $4 \AA$ for emission from the VB and $2 p$ CL band, respectively) and NIR skin depth $\left(\delta_{L}=2 \AA\right)$ are in accordance with the MFPs suggested in Refs. [4,34,38] and the skin depth for free-electron metals calculated in Ref. [39]. The agreement between our quantum-mechanical and the experimental relative streaking time delay [4] gives us confidence in applying the Chulkov and Chulkov-Yukawa initial-state models, together with carefully determined values of the MFP and NIR skin depth, to future investigations of the dependence of streaked photoemission spectra on the substrate properties (and thickness of adsorbate layers) of different (adsorbate-covered) surfaces.

\section{ACKNOWLEDGMENTS}

This work was supported by NSF Grant No. PHY 1068752 and by the Chemical Sciences, Geosciences, and Biosciences Division, Office of Basic Energy Sciences, Office of Science, US DOE.
[1] M. Schultze, M. Fieß, N. Karpowicz, J. Gagnon, M. Korbman, M. Hofstetter, S. Neppl, A. L. Cavalieri, Y. Komninos, Th. Mercouris, C. A. Nicolaides, R. Pazourek, S. Nagele, J. Feist, J. Burgdörfer, A. M. Azzeer, R. Ernstorfer, R. Kienberger, U. Kleineberg, E. Goulielmakis, F. Krausz, and V. S. Yakovlev, Science 328, 1658 (2010).

[2] K. Klünder, J. M. Dahlström, M. Gisselbrecht, T. Fordell, M. Swoboda, D. Guénot, P. Johnsson, J. Caillat, J. Mauritsson, A. Maquet, R. Taïeb, and A. L'Huillier, Phys. Rev. Lett. 106, 143002 (2011).
[3] A. L. Cavalieri, N. Müller, Th. Uphues, V. S. Yakovlev, A. Baltuška, B. Horvath, B. Schmidt, L. Blümel, R. Holzwarth, S. Hendel, M. Drescher, U. Kleineberg, P. M. Echenique, R. Kienberger, F. Krausz, and U. Heinzmann, Nature (London) 449, 1029 (2007).

[4] S. Neppl, R. Ernstorfer, E. M. Bothschafter, A. L. Cavalieri, D. Menzel, J. V. Barth, F. Krausz, R. Kienberger, and P. Feulner, Phys. Rev. Lett. 109, 087401 (2012).

[5] M. Hentschel, R. Kienberger, Ch. Spielmann, G. A. Reider, N. Milosevic, T. Brabec, P. Corkum, U. Heinzmann, 
M. Drescher, and F. Krausz, Nature (London) 414, 509 (2001).

[6] F. Quéré, Y. Mairesse, and J. Itatani, J. Mod. Opt. 52, 339 (2005).

[7] V. S. Yakovlev, F. Bammer, and A. Scrinzi, J. Mod. Opt. 52, 395 (2005).

[8] U. Thumm, Q. Liao, E. M. Bothschafter, F. Süßmann, M. F. Kling, and R. Kienberger, Attosecond Physics: Attosecond Streaking Spectroscopy of Atoms and Solids, Handbook of Photonics (Wiley, New York, in press).

[9] A. S. Kheifets and I. A. Ivanov, Phys. Rev. Lett. 105, 233002 (2010).

[10] L. R. Moore, M. A. Lysaght, J. S. Parker, H. W. van der Hart, and K. T. Taylor, Phys. Rev. A 84, 061404 (2011).

[11] J. M. Dahlström, T. Carette, and E. Lindroth, Phys. Rev. A 86, 061402 (2012).

[12] A. S. Kheifets, Phys. Rev. A 87, 063404 (2013).

[13] S. Nagele, R. Pazourek, J. Feist, and J. Burgdörfer, Phys. Rev. A 85, 033401 (2012).

[14] E. E. Krasovskii, Phys. Rev. B 84, 195106 (2011).

[15] C. A. Rios Rubiano, M. S. Gravielle, D. M. Mitnik, and V. M. Silkin, Phys. Rev. A 85, 043422 (2012).

[16] C. Lemell, B. Solleder, K. Tőkési, and J. Burgdörfer, Phys. Rev. A 79, 062901 (2009).

[17] A. K. Kazansky and P. M. Echenique, Phys. Rev. Lett. 102, 177401 (2009).

[18] C.-H. Zhang and U. Thumm, Phys. Rev. Lett. 102, 123601 (2009); 103, 239902(E) (2009).

[19] A. K. Kazansky and P. M. Echenique, Phys. Rev. B 81, 193413 (2010).

[20] C.-H. Zhang and U. Thumm, Phys. Rev. A 84, 063403 (2011).

[21] E. V. Chulkov, V. M. Silkin, and P. M. Echenique, Surf. Sci. 437, 330 (1999).
[22] Q. Liao and U. Thumm, Phys. Rev. Lett. 112, 023602 (2014).

[23] U. Thumm, J. Phys. B 25, 421 (1992); P. Kürpick, U. Thumm, and U. Wille, Phys. Rev. A 56, 543 (1997).

[24] J. C. Baggesen and L. B. Madsen, Phys. Rev. A 78, 032903 (2008).

[25] C.-H. Zhang and U. Thumm, Phys. Rev. A 80, 032902 (2009).

[26] C.-H. Zhang and U. Thumm, Phys. Rev. A 84, 065403 (2011).

[27] S. Neppl, Ph.D. thesis, Technische Universität München, 2012.

[28] A. G. Borisov, D. Sánchez-Portal, A. K. Kazansky, and P. M. Echenique, Phys. Rev. B 87, 121110(R) (2013).

[29] N. W. Ashcroft and N. D. Mermin, Solid State Physics (Saunders College, Philadelphia, 1976).

[30] G. F. Gribakin and M. Yu. Kuchiev, Phys. Rev. A 55, 3760 (1997).

[31] C.-H. Zhang and U. Thumm, Phys. Rev. A 82, 043405 (2010).

[32] D. Bauer, D. B. Milošević, and W. Becker, Phys. Rev. A 72, 023415 (2005).

[33] B. H. Bransden and C. J. Joachain, Physics of Atoms and Molecules, 2nd ed. (Prentice-Hall, Englewood Cliffs, NJ, 2003).

[34] A. Zangwill, Physics at Surfaces (Cambridge University Press, New York, 1988).

[35] S. Hüfner, Photoelectron Spectroscopy, 3rd ed. (Springer, Berlin, 2003).

[36] E. V. Chulkov and V. M. Silkin, Solid State Commun. 58, 273 (1986); E. V. Chulkov, V. M. Silkin, and E. N. Shirykalov, Surf. Sci. 188, 287 (1987).

[37] R. A. Bartynski, R. H. Gaylord, T. Gustafsson, and E. W. Plummer, Phys. Rev. B 33, 3644 (1986).

[38] S. Tanuma, C. J. Powell, and D. R. Penn, Surf. Interface Anal. 43, 689 (2011).

[39] E. E. Krasovskii, V. M. Silkin, V. U. Nazarov, P. M. Echenique, and E. V. Chulkov, Phys. Rev. B 82, 125102 (2010). 